\title{
Effect of Ocular Dominance on the Latency and Amplitude of Visual Evoked Potentials
}

\author{
${ }^{1}$ Jagadamba .A, ${ }^{2}$ Karthiyanee kutty \\ ${ }^{1,2}$ Department of Physiology,Sri Devaraj Urs Medical College, Tamaka, Kolar
}

\begin{abstract}
Background: Ocular dominance is the tendency to prefer visual input from one eye to the other. $V E P$ s are the electrical potentials recorded from the scalp in response to visual stimuli to assess conduction pathway from the retina to the occipital cortex.
\end{abstract}

Objectives: 1. to determine ocular dominance.

2. To compare the latency and amplitude of Visual evoked potentials in dominant and non dominant eye.

Materials \& methods: Among 100 normal healthy subjects, Ocular dominance was determined by Miles \& Porta test \& then VEPs was recorded. Latency \& amplitude of individual VEP wave parameters were measured.

Results: Eye dominance by Porta \& Miles test, showed Right eye dominant were $75 \%$ \& left eye dominant 25\%. Eye dominance on Amplitude is increased in dominant eye which is statistically significant compared to the non-dominant eye. Eye dominance on latency of N70 and P100 is decreased in dominant eye which is statistically significant compared to the non-dominant eye.

Conclusion: The latency of $N 70$ \& $P 100$ has been found to be lesser in the dominant eye and the amplitude is increased in the dominant eye as compared to non-dominant eye, which provides electrophysiological evidence of lateralization in the central nervous system.

Key words: Visual evoked potentials, ocular dominance

\section{Introduction}

Dominance of one member of a bilateral pair of bodily structures means a functional priority, superiority, or preference of that member. Functional lateralization occurs in the paired organs of the body, such as hands, legs, and cerebral hemispheres. Ocular dominance was first described in 1953 by Giovanni Battista Porta. Ocular dominance, otherwise called eye dominance or eyedness was the tendency to prefer visual input from one eye to the other $[7,13]$. The eye is a sensory organ and has no conscious proprioception and vision in each eye is represented bilaterally and equally in the occipital lobes. Individuals have no consciousness of using right or left eye, as one is conscious of having left or right hand. An individual does not see the world from left or right eye but from a single so called cyclopean eye, which combines information from both. Dominance wise eyes work as ones hand. They grab the image with one eye and pass on to other and start to analyze the object by refining like using their fingertips or balancing objects with two hands [1].

Around two-thirds of the population is right eye dominant, however neither eye is dominant in a small portion of the population [2.3]. Dominance does appear to change depending upon direction of gaze due to image size changes on the retinas [4]. Furthermore, the eye preferred for sighting does not indicate handedness. This is not surprising since each eye projects to both cerebral hemispheres whereas each hand is represented mainly in the opposite hemisphere.

In normal binocular vision there is an effect of parallax, and therefore the dominant eye is the one that is primarily relied on for precise positional information. This may be particularly important in sports which require aim, such as Archery, darts or shooting sports [5]. Ocular dominance is of great magnitude in predicting patient satisfaction with mono vision correction in cataract surgery, refractive surgery and contact lens wear [6].

The dominant eye has higher degree of myopic refractive error and longer axial length than the non dominant eye especially in patients with high amounts of anisometropia[14].

The repeated suppression of the non sighting eye to avoid physiologic diplopia and the weaker motoric drive may predispose the non-dominant eye to amblyopia ex anopsia [8].

A directional dominance or sighting test can be done in various ways. For example, a patient will form a hole with his hands and binocularly center an object in that hole. When he alternately occludes either eye, only the dominant eye will still see the same object [10].

The VEP assess the functional integrity of the visual pathway from the receptors in the retina to the occipital cortex. It measures the conduction of the visual pathways from the optic nerve, optic chiasm and optic radiations to the occipital cortex. The most important fact to consider is that although the axons form the nasal half of the retina decussate at the optic chiasm, the temporal axons do not. Therefore, retrochiasmatic lesions 
may not be detected by full-field checkerboard stimulation. VEPs are most useful in testing optic nerve function and less useful in postchiasmatic disorders [9].

The VEPs has been used to study various physiological parameters, very few studies have been done to compare the ocular dominance with visual evoked potentials.

So the aim of study is to determine ocular dominance and its effect on the latency and amplitude components of Visual evoked potentials in dominant and non dominant eye in normal healthy subjects.

\section{Materials And Methods}

The Study group consisted of 100 normal healthy subjects aged 18-40 years with normal vision6/6 with or without glasses who volunteered. Ethical clearance was obtained from Institutional Ethics Committee. Informed consent was obtained. The exclusion criteria were subjects with visual impairment, history of use of drug intake (mydriatics \& myotics) \& history of any chronic medical illness.

\subsection{Methodology}

During phase of the experiment, subjects were screened as per the criteria laid down under inclusion \& exclusion criteria. The informed consent was taken from selected subjects \& they were given specific dates to come to neurophysiology lab and hair washed without applying oil.

Data was collected after clinical examination visual acuity of $6 / 6$ with normal visual fields was ensured. On entering lab the subjects were explained about the procedure. Ocular dominance was determined by Miles test and confirmed by Porta test and then the visual evoked potentials were recorded.

\subsection{Miles Test}

In this test the observer extends both arms, brings both hands together to create a small opening, then with both eyes open views a distant object through the opening. The observer then alternates closing the eyes or slowly draws opening back to the head to determine which eye is viewing the object, that is the dominant eye [12].

\subsection{Porta Test}

The observer extends one arm, and then with both eyes open aligns the thumb or index finger with a distant object. The observer than alternates closing the eyes or slowly draws the thumb/finger back to the head to determine which eye is viewing the object ,that is the dominant eye [10].

Visual Evoked Potentials were recorded using RMS-EMG MARK-II machine. Visual stimulus was presented to the subject 200 times on computer screen at a distance of $100 \mathrm{~cm}$, with checker pattern of size $8 \times 8$.

The most important step in recording VEP was fixing of electrode. The electrode was fixed as per 1020 system which was originally devised for EEG recording. Using the 10-20 system the reference midfrontal electrode was placed at $30 \%$ and recording electrodes at $10 \%$ of the whole distance from nasion to occipital protuberance. Thus reference electrode was placed at $10-13 \mathrm{~cm}$ above nasion and recording electrodes at $3-5 \mathrm{~cm}$ above the occipital protuberance (inion).

The electrodes are attached to the surface of scalp with conducting paste after cleaning vigorously keeping skin resistance as low as $5 \mathrm{k} \Omega$ and these electrodes were connected to electrode box by connecting wires.

One eye was selected, with other eye closed with mask. The subject was asked to see on the fixation point on the center of screen, relaxed, blinking minimally and concentrating on the stimulus pattern. The checks were made to reverse at a rate of $1 \mathrm{~Hz}$ and 200 responses were recorded. The waveform latency in msec and amplitude in $\mu$ volts were measured inbuilt.

\section{Statistical Analysis}

Descriptive statistical analysis was carried out on this data in the present study. Results on continuous measurements were presented as Mean \pm SD (Min-Max) and results on categorical measurements were presented in Number (\%). Significance was assessed at $5 \%$ level of significance. Student t test (two tailed, dependent) has been used to find the significance of study parameters on continuous scale within each group. Percentile distribution of latencies and amplitude in dominant and non dominant eye were computed.

\subsection{Results}

Table 1 provides information about the comparision of eye dominance by Porta \& Miles test, where Right eye dominant were $75 \%$ \& left eye dominant $25 \%$ in both Portas \& Miles test. In this study we encountered no subject with conflicting results from the Portas \& Miles test. 
Table 2 Shows amplitude of dominant eye with a minimum value of $8.06 \mu \mathrm{v} \&$ a maximum value of $11.50 \mu \mathrm{v}$ with a mean value of $9.88 \pm \mathrm{SD}$ of 0.79 . Non dominant eye showed a minimum value of $7.57 \mu \mathrm{v} \& \mathrm{a}$ maximum value of $10.89 \mu$ v with a mean value of $9.45 \pm$ SD of 0.73 . (Graph 8)

Table 3 Shows dominant eye latency of $\mathrm{N} 70$ with a minimum value of $56.25 \mathrm{~ms} \&$ a maximum value of $80.60 \mathrm{~ms}$ with a mean value of $65.27 \pm \mathrm{SD}$ of 5.67. Dominant eye P100 showed a minimum value of $80.00 \mathrm{~ms}$ \& a maximum value of $104.38 \mathrm{~ms}$ with a mean value of $90.73 \pm$ SD of 7.34.Dominant eye N155 showed a minimum value of $143.75 \mathrm{~ms} \&$ a maximum value of $181.25 \mathrm{~ms}$ with a mean value of $161.47 \pm \mathrm{SD}$ of 10.20 .

Non dominant eye latency N70 showed a minimum value of $58.80 \mathrm{~ms} \&$ a maximum value of $80.60 \mathrm{~ms}$ with a mean value of $65.91 \pm$ SD of 5.40. Non dominant eye latency P100 showed a minimum value of $80.00 \mathrm{~ms}$ $\&$ a maximum value of $119.40 \mathrm{~ms}$ with a mean value of $94.29 \pm \mathrm{SD}$ of 8.22 . Non dominant eye latency N155 showed a minimum value of $123.75 \mathrm{~ms} \&$ a maximum value of $184.38 \mathrm{~ms}$ with a mean value of $160.60 \pm$ SD of 12.30.Also shows the percentile distribution of these measures. (Graph 4)

Table 4 shows the effect of eye dominance on Amplitude is increased in dominant eye which is statistically significant $(\mathrm{P}<0.001)$ compared to the non-dominant eye .(Graph 3$)$

The effect of eye dominance on latency of N70 and P100 is decreased in dominant eye which is statistically significant ( P $0.006 \&<0.001$ ) compared to the non-dominant eye . (Graph $4 \& 5$ )

The results of $\mathrm{N} 155$ was not statistically significant in the dominant and nondominant eye.(P 0.353 ).(Graph 6)

\section{Tables And Graphs}

Table 1:

Comparison of eye dominance by Porta \& Miles test.

\begin{tabular}{|l|l|l|l|}
\hline Eye dominance & Right eye & Left eye & Total \\
\hline Miles Test & 75 & 25 & 100 \\
\hline Porta Test & 75 & 25 & 100 \\
\hline Total & 150 & 50 & 200 \\
\hline
\end{tabular}

Table 2: Percentile distribution of amplitude Visual evoked Potentials of $\mu \mathrm{v}$ in dominant and non-dominant eye in young adults

\begin{tabular}{|c|c|c|c|c|c|c|c|c|c|}
\hline \multirow{2}{*}{ VEP } & \multirow{2}{*}{ Min } & \multirow{2}{*}{ Max } & \multirow{2}{*}{ Mean } & \multirow{2}{*}{ SD } & \multicolumn{5}{|c|}{ Percentile } \\
\cline { 6 - 10 } & & & & $\mathbf{5}^{\text {th }}$ & $\mathbf{2 5}^{\text {th }}$ & $\mathbf{5 0}^{\text {th }}$ & $\mathbf{7 5}^{\text {th }}$ & $\mathbf{9 5}^{\text {th }}$ \\
\hline dominant eye & 8.06 & 11.50 & 9.88 & 0.79 & 8.48 & 9.42 & 9.76 & 10.65 & 11.10 \\
\hline $\begin{array}{c}\text { Non dominant } \\
\text { eye }\end{array}$ & 7.57 & 10.89 & 9.45 & 0.73 & 8.19 & 9.06 & 9.43 & 9.72 & 10.71 \\
\hline
\end{tabular}

Graph 1:

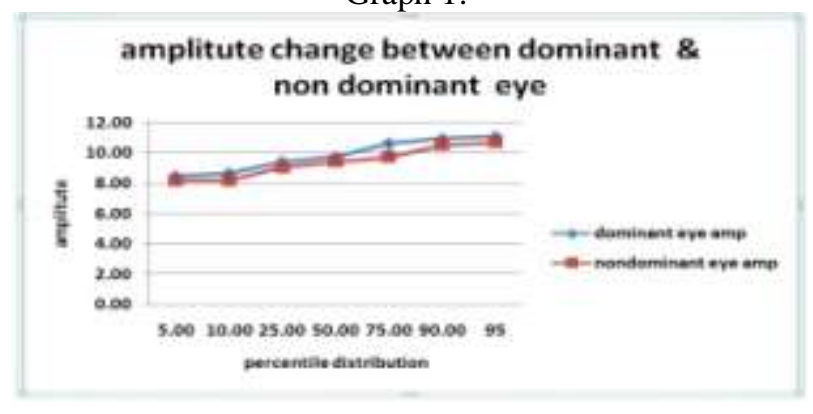

Table 3 :Percentile distribution of latencies of Visual evoked Potentials in dominant and non-dominant eye in young adults

\begin{tabular}{|c|c|c|c|c|c|c|c|c|c|c|}
\hline \\
\hline & & \multirow{3}{*}{$\frac{\text { Min }}{56.25}$} & \multirow[b]{2}{*}{$\operatorname{Max}$} & \multirow[b]{2}{*}{ Mean } & \multirow[b]{2}{*}{ SD } & \multicolumn{5}{|c|}{ Percentile } \\
\hline \multicolumn{2}{|c|}{ Latencies } & & & & & $5^{\text {th }}$ & $25^{\text {th }}$ & $50^{\text {th }}$ & $75^{\text {th }}$ & $95^{\text {th }}$ \\
\hline \multirow{3}{*}{$\begin{array}{c}\text { Dominant } \\
\text { eye }\end{array}$} & N70 & & 80.60 & 65.27 & 5.67 & 60.00 & 62.50 & 63.10 & 66.30 & 80.60 \\
\hline & P100 & 80.00 & 104.38 & 90.73 & 7.34 & 80.00 & 83.80 & 91.35 & 97.88 & 101.30 \\
\hline & N155 & 143.75 & 181.25 & 161.47 & 10.20 & 149.40 & 151.90 & 162.50 & 169.40 & 180.60 \\
\hline \multirow{3}{*}{$\begin{array}{c}\text { non } \\
\text { dominant } \\
\text { eye }\end{array}$} & N70 & 58.80 & 80.60 & 65.91 & 5.40 & 61.36 & 63.10 & 63.80 & 67.30 & 80.59 \\
\hline & P100 & 80.00 & 119.40 & 94.29 & 8.22 & 80.00 & 86.30 & 95.70 & 101.30 & 101.90 \\
\hline & N155 & 123.75 & 184.38 & 160.60 & 12.30 & 145.66 & 151.90 & 158.40 & 169.40 & 180.60 \\
\hline
\end{tabular}


Graph 2:

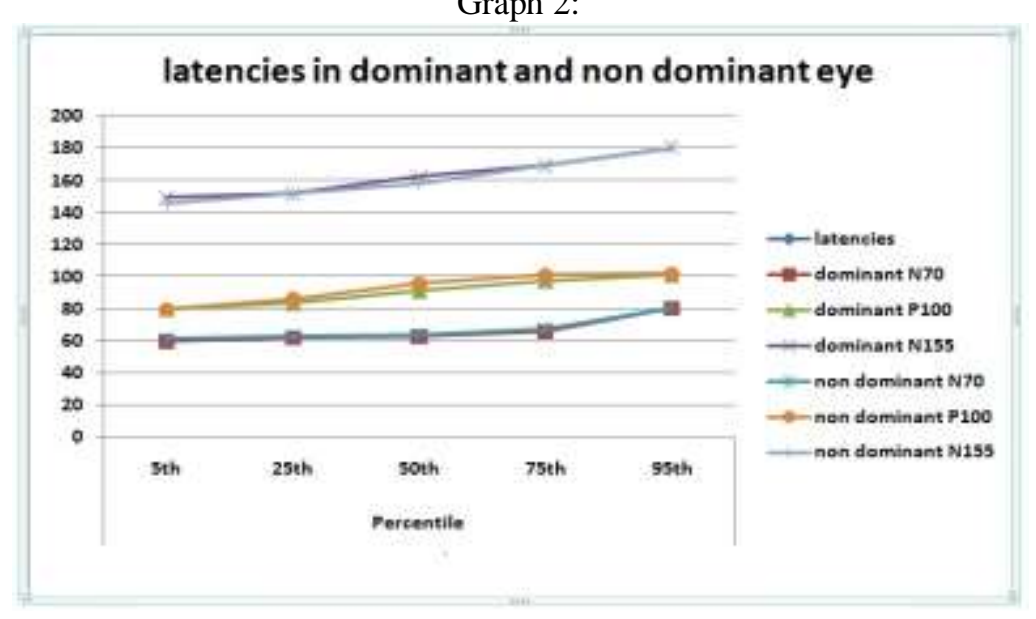

Table 4: Comparison of amplitude and Latency in Dominant and Non-dominant eyes

\begin{tabular}{|l|c|c|c|}
\hline & Dominant eyes & $\begin{array}{c}\text { Non-Dominant } \\
\text { eyes }\end{array}$ & P value \\
\hline Amplitude $(\mu \mathrm{v})$ & $9.88 \pm 0.79$ & $9.45 \pm 0.73$ & $<0.001^{* *}$ \\
\hline N70 & $65.27 \pm 5.67$ & $65.91 \pm 5.40$ & $0.006^{* *}$ \\
\hline P100 & $90.73 \pm 7.34$ & $94.29 \pm 8.22$ & $<0.001^{* *}$ \\
\hline N155 & $161.47 \pm 10.20$ & $160.6 \pm 12.3$ & 0.353 \\
\hline
\end{tabular}

Graph 3: Comparison of amplitude in Dominant and Non-dominant eyes

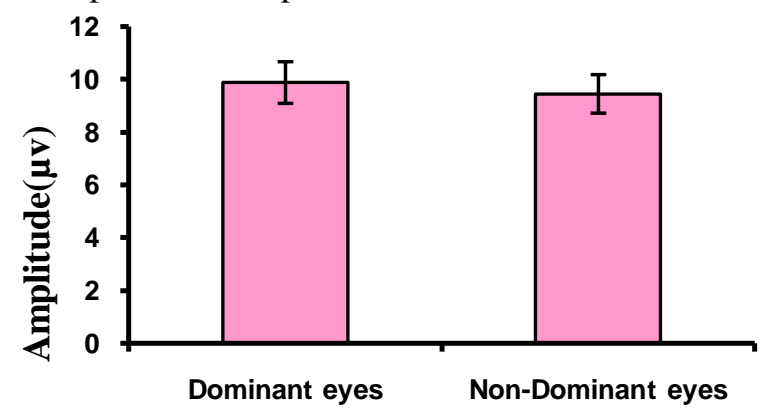

Graph 4: Comparison of N70 latency in Dominant and Non-dominant eyes

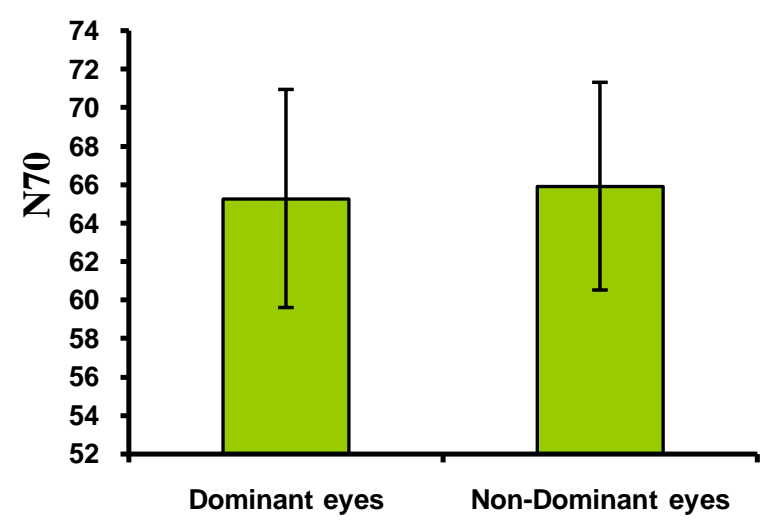

Graph 5: Comparison of P100 latency in Dominant and Non-dominant eyes 


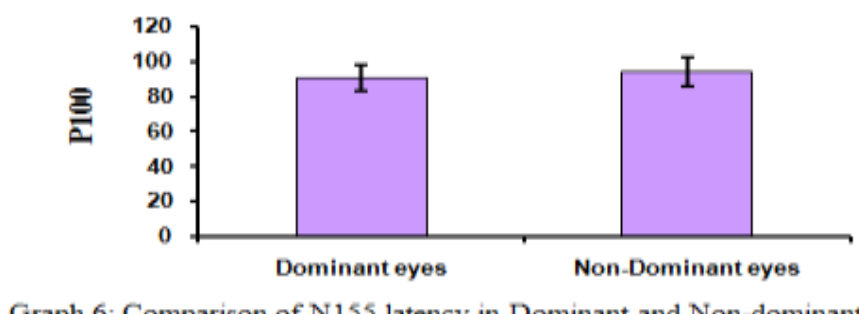

Graph 6: Comparison of N1S5 latency in Dominant and Non-dominant eyes

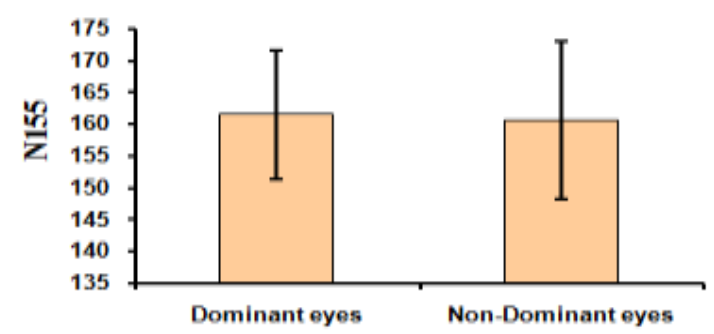

\section{Discussion}

Dominance is mainly influenced by genetics and is defined as physiological priority or preference by one member of any bilateral pair of structures in the body when performing various tasks [12]. The lateralization in eye function is the result of development of binocular vision, with overlapping of the visual fields of the two eyes. The resulting physiologic diplopia is suppressed from the non-sighting eye. This is thought to be the mechanism of development of ocular dominance. The input is favoured from the sighting eye. When a choice is forced between the two eyes, the vast majority of people choose one eye consistently [8].

Some authors have commented that laterality dominance in terms of eyedness is an obvious phenomenon, although its functional significance is not clear. Some studies have indicated that $60 \%$ of their sample preferred right eye [14]. In one study, 54 patients with either unilateral ptosis or asymmetrical ptosis were examined. The right eye was dominant in 33 people and the left in 21people [17]. Ptosis occurred on the side of the dominant eye in 14 individuals and on the non-dominant side in 40 individuals. The study suggests that levator muscle tone is influenced by eye dominance when ptosis is present. It may be important to treat first the non-dominant eye in ptosis, which could look worse than it really is if it affects the dominant eye as a result of retraction of the contralateral levator muscle [17].

Approximately $2 / 3$ of the population is right eye dominant. In our study, by sighting dominance we found that $75 \%$ were right eye dominant \& $25 \%$ were left eye dominant, which was consistent with the results of one more study [14]. In our study no subjects were encountered with conflicting results from the Portas \& Miles test. Sighting dominance is most important in clinical investigations \& most clinical phenomenon are related to this type of dominance in ptosis, cataract, myopia etc $[8,14,17]$.

Visual evoked potentials are the electrical potentials evoked in the brain by visual stimuli and measure the speed of the visual pathways and also the synchronized electrical activity in response to a visual stimulus. In our study, we have measured the activity of monocular stimulation in normal subjects, and have observed the differences between the amounts of activity evoked by each eye. These values are compared with sighting eye dominance (DE\&NDE). It was revealed in a research that using positron emission test and VEP, integration of visual information begins in the fusiform gyrus as soon as 80 to 130 milli seconds after the visual stimulus in a normal human [15].

In our study the time taken from the stimulus to wave N70 of the visual pathway was shorter in dominant eye compared to the non dominant eye which is statistically significant. Thus a shorter N70 latency represents faster visual processing in the dominant eye. In a study it was shown that significant visual processing occurs within $150 \mathrm{~ms}$ after ball release in sports and all the main features of VEP also occurs within a $150 \mathrm{~ms}$ period. A shorter N70 latency therefore represents faster visual processing which is required immediately following ball release in skilled batsmen and would become more critical with high speed deliveries [16].

In our study the time taken from the stimulus to wave P100 latency was also faster in dominant eye, reflecting the activity of visual cortex. In another study it was found that there is a faster P100 latency $(\sim 4 \mathrm{~ms})$ in tennis and squash players compared to rowers and sedentary subjects. They related the faster P100 latency, which reflects activity of the visual cortex to a tennis player's ability to rapidly process sensory information [18]. Data of a study showed that, the mean latency of P100 peak was significantly shorter with stimulation of the dominant eye and amplitude were higher in the dominant eye, which provides objective electrophysiological evidence of lateralization in the central nervous system [11]. 
Another study revealed that the influence of eye dominance scaled by six tests on the parameters (N80, P100latency and N80-P100 amplitude) of the white-black, green-black, red-black and blue-black pattern visual evoked potentials, with normal visual acuity. The P100 latency of the white black PVEPS, for both sexes, significantly shorter in the PVEPs of the dominant eye. The results given were that further electrophysiological evidence for eye dominance as a lateralized CNS phenomenon is not influenced by colour [19].

In our study the Amplitudes were higher in recordings of V. E. P from dominant eye than from the nondominant eye which is statistically significant. This is because of increased electrical activity in the visual pathway.This is in accordance in a study, that showed the visual processing during the first $100-150 \mathrm{~ms}$ of the ball flight with binocular vision facilitates retinal activation in talented cricketers [20]. On the other hand, one study performed a spectral analysis on steady state V. E. P obtained from dominant and non-dominant eyes found no difference in power values of VEP [21]. Another study reported that eye dominance of dextrals appeared to play a role in determining the hemispheric asymmetry [22].

VEP is very important non-invasive tool in detecting abnormalities of visual system \& have a wide range of clinical applications. As technology continues to evolve, VEP will likely provide more qualitative and quantitative information regarding the function of the optic nerve \& kinetics of synaptic plasticity. Study of normal ocular dominance is amenable for future studies with VEP using variety of stimuli and such physiological measures are needed to fully understand the functional significance.

It is not only useful for clinical neurophysiologist or ophthalmologist but also for neurologist and neurosurgeons. Sighting dominance is most important in clinical investigations.

In addition, understanding the extent of eye dominance in normal subjects is important for the study and interpretation of monocular clinical eye diseases (ptosis, amblyopia, myopia etc.) and also in sports which require aim (archery, darts or shooting).

\section{Conclusion}

In conclusion, $75 \%$ of the subjects were right eye dominant and $25 \%$ were left eye dominant. The latency of N70 \& P100 has been found to be lesser in the dominant eye and the amplitude is increased in the dominant eye as compared to non-dominant eye, which provides electrophysiological evidence of lateralization in the central nervous system.

\section{Acknowledgements}

Prof.Ravi.M, Dept of Anaesthesiology for helping in editing and constant support.

\section{References}

[1] Sharp-sighted.org [homepage on Internet]. Brussels: Vandegaer M ;[updated 2009]. Facial masking, Alignment \& Dyslexia.0.3 Ocular Dominance; [about 12 screens]. Available from: http://www.sharp-sighted.org

[2] Ehrenstein WH, Gahmen BE, Jaschinski W. Eye preference within the context of binocular functions. Graefes Arch Clin Exp Ophthalmol 2005 Sep; 243(9):926-932.

[3] Reiss MR.Ocular dominance: some family data. Laterality 1997; 2(1):7-16.

[4] Khan AZ, Crawford JD. Ocular dominance reverses as a function of horizontal gaze angle. Vision Res 2001; 41(14):1743-1748.

[5] Siviero MO, Rysovas EO, Juliano Y, DelPorto JA, Bertolucci PHF. Eye-hand preference dissociation in obsessive-compulsive disorder and dyslexia. Arg Neuropsiquiatr 2002; 60(2-A):242-245.

[6] Connolly BH. Lateral dominance in children with learning disabilities. Phys Ther 1983; 63(2):183-187.

[7] Chaurasia BD, Mathur BB. Eyedness. Acta Anat 1976; 96(2):301-305.

[8] Hashim AA. Eye Dominance in Excimer Laser Photorefractive Keratectomy. J Refract Surg 1997; 13: 461 - 462.

[9] Lecture 11- Ocular Dominance(Steinman Chapter 2, p. 24-27; Chapter 3, p. 56-57)

[10] Mendola JD, Conner IP. Eye dominance predicts fMRI signals in retinotopic cortex. Neurosci Lett 2007; 414: $30-34$

[11] Seyal M, Sato S, White BG. Visual evoked potentials and eye dominance. Electroencephalogr Clin Neurophysiol 1981;52:424.

[12] Brackenridge CJ. The contribution of genetic factors to ocular dominance. Behav Genet 1982; 12(3):319-325.

[13] Khan AZ, Crawford JD. Ocular dominance reverses as a function of horizontal gaze angle. Vision Res 2001; 41(14):1743-8.

[14] Cheng CY, Yen MY, Lin HY, Hsia WW, Hsu WM. Association of ocular dominance and anisometropic myopia. Invest Ophthalmol Visual Sci 2004;45(8):2856-2860.

[15] Heinze HJ, Mangun G, Burchert W, Bodino G, Gastaud M. Combined spatial and temporal imaging of brain activity during visual selective attention in humans. Nature 2002; 372, 543-546.

[16] Land M, McLeod P. From eye movement to actions: how batsmen hit the ball. Nat Neurosci 2000;3:1340-45.

[17] Lyon DB, Gonnering RS, Dortzbach RK, Lemke BN. Unilateral ptosis and eye dominance. Ophthal Plast Reconstr Surg 1993; 9(4):237-40.

[18] Delpont E, Dolisi C, Suisse G, Bodino G, Gastaud M. Visual evoked potentials: differences related to physical activity. Int J Sports Med 1991;12(3):293-8.

[19] Taghavy A, Kugler CF. A Pattern Reversal Visual Evoked Potentials in Study of eye dominance. Eur Arch Psychiatr Neurol Sci 1987; 236: 329-332.

[20] Thomas NG, Harden LM, Rogers GG. Visual Evoked Potentials,reaction times and eye dominance in cricketers. J Sports Med Phys Fitness.2005;45(3):428-33.

[21] Klemm WR, Gibons WD, Allen RG, Richey EO. Hemispheric lateralization and handness correlation of human evoked steady state responses to patterned visual stimuli. Physiol Psycholl 1980; 8:409-416.

[22] Spinelli D, Mecacci L. Handedness and hemispheric asymmetry of pattern reversal visual-evoked potentials. Brain Cogn 1990;13(2):193-210. 